\title{
Una fórmula para alcanzar la sostenibilidad empresarial
}

\section{A formula to achieve business sustainabilitity}

\section{Valentina Cárdenas Cadena ${ }^{1}$}

(2).

Recepción: 20/09/2020
()...

Aprobación: 04/11/2020
-

Publicación: 18/12/2020

Para citar este artículo:

Cárdenas Cadena, V. (2020). Una fórmula para alcanzar la sostenibilidad empresarial. Indagare, (8), 247-253. https://doi.org/10.35707/indagare/823

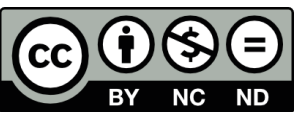

${ }^{1}$ Estudiante del programa de Comunicación Social y Periodismo de la Universidad de Ibagué. ORCID: 0000-0001-85696745. Correo electrónico: cardenas.valentina0@gmail.com 


\title{
Resumen
}

La creatividad y el ingenio son factores claves que desarrollan las áreas de gestión humana de una empresa, para que sus trabajadores generen un sentido de pertenencia por su organización. Existen diversas prácticas que procuran pensar en el bienestar del empleador y en el desarrollo de la entidad, esta idea es producto de una investigación que llevaron a cabo la Universidad de Ibagué y el Instituto Tecnológico de Monterrey que otorga como resultado la publicación del libro Prácticas sostenibles de Gestión Humana. Un énfasis desde la Responsabilidad Social Interna en 2020.

\begin{abstract}
Creativity and ingenuity are key factors that human resources areas of a company develop, so that its employees generate a sense of belonging to their organization. There are various practices that try to think about the employer and the development of the entity; this idea is the product of a research carried out by Universidad de Ibague and the Technological Institute of Monterrey that results in the publication of the book Sustainable Practices of Human Resources. An emphasis from the Internal Social Responsibility in 2020.
\end{abstract}

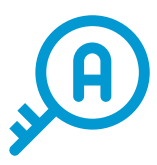

Palabras claves: Responsabilidad social, gestión humana, empresas, clima organizacional, prácticas sostenibles.

Key words: Social responsibility, human resources, companies, corporate environment, sustainable practices

Cada área de una empresa tiene funciones específicas que permiten el buen funcionamiento de la organización. Pero además de esto, se vinculan valores de liderazgo y compromiso con los cuales deben contar los empleadores para lograr no solo un buen rendimiento en las funciones administrativas, sino también un clima organizacional saludable. Sin embargo, este aspecto no es solo responsabilidad de los empleadores en su diario vivir con la empresa. Existen dos dependencias que fueron creadas con el propósito de generar sentido de pertenencia en los trabajadores por su organización: las áreas de gestión humana y de responsabilidad social. La pregunta es ¿cómo logran hacer esto?

Estas son algunas ideas que permitieron la consolidación del libro Prácticas sostenibles de Gestión Humana. Un énfasis desde la Responsabilidad Social Interna en el año 2020, dirigido por las docentes Alba Ruth Vargas Montealegre, de la Universidad de Ibagué, y Cecilia Eugenia Valdez Gutiérrez, del Instituto Tecnológico de Monterrey. 
Indagare tuvo la oportunidad de conversar con Alba Ruth Vargas Montealegre, quien es administradora de empresas, especialista en Gerencia del Talento Humano y Desarrollo Organizacional, magíster en Gerencia del Talento Humano y magíster en Educación. Lleva diez años vinculada con la Universidad de Ibagué como docente de tiempo completo del programa de Administración de Empresas de la Facultad de Ciencias Económicas y Administrativas.

Figura 1. Retrato de

Alba Ruth Vargas Montealegre

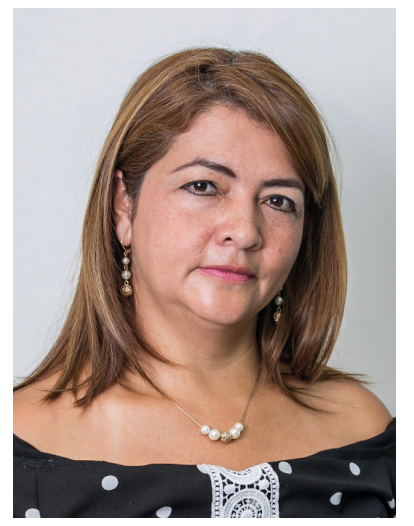

"La gestión humana orientada a cumplir la responsabilidad social interna exige una mirada real de reconocimiento al trato digno del ser humano y a su labor en las organizaciones como el que moviliza todos los recursos para alcanzar un máximo desempeño, innovación y desarrollo" (A. Montealegre, comunicación telefónica, 4 de septiembre de 2020).

Fuente: Comunicación institucional, Universidad de Ibagué

\section{¿Cómo nace el libro y logran vincularse con México?}

Venía trabajando temas de investigación en gestión humana y empezamos a notar que, al vincular a las personas con la responsabilidad social, se podría contribuir al desempeño tanto de las empresas como de los grupos de interés. El enfoque que queríamos trabajar era centrado en la responsabilidad social de las personas para las personas y la contribución que se logra desarrollar en las empresas, como una ganancia desde diferentes puntos de vista. Se presentó la oportunidad de trabajar con la profesora Cecilia Valdez del Instituto Tecnológico de Monterrey y ella aceptó la invitación.

En la investigación se vincularon estudiantes y personas que desarrollaban su labor en actividades de responsabilidad social en México. En Colombia se formó un semillero de investigación en la Universidad de Ibagué con estudiantes de la Facultad de 
Ciencias Económicas y Administrativas del programa de Administración de Negocios Internacionales. También participaron estudiantes de Ingeniería Industrial y Psicología y contamos con el apoyo y la orientación del equipo de Ediciones Unibagué para la publicación del libro.

¿Qué tan productivo fue ese intercambio de experiencias no solo con diferentes disciplinas sino con otro país?

Una ventaja que tuvimos fue que la investigación se estructuró dentro del marco de un programa de la Universidad de Ibagué: la Escuela Internacional de Verano. Allí fue donde nos unimos y estructuramos un equipo de trabajo fuerte, que generó conocimientos mutuos y experiencias importantes que se transfirieron a los estudiantes y permitieron una experiencia académica muy enriquecedora y significativa para ambas instituciones.

¿Por qué se suele separar las prácticas de gestión humana y responsabilidad social, y por qué deberían estar unidas?

Generalmente, notamos en el estudio que algunas organizaciones, según sus capacidades y su tamaño, han creado dos áreas: una de responsabilidad social y otra de gestión humana. En esas empresas en las que no hay una interconexión de estas dos dependencias, se hacen actividades importantes, pero no redundan en la cultura organizacional. En las empresas en donde sí están conectadas, se genera una cultura que produce beneficios para el desarrollo de la organización y sus colaboradores.

¿Hay alguna diferencia cultural para el establecimiento de las prácticas de gestión humana?

Sí, la cultura es un tema muy amplio y que hace parte de la identidad de cada organización. Las empresas tienen su cultura, que es el conjunto de valores, símbolos y reconocimientos compartidos, que marcan la esencia de la organización. Esa visión en conjunto es diferente de una organización a otra, pero conserva similitudes en su contexto. Por ejemplo, en Latinoamérica se viven realidades como las barreras tributarias, la precariedad del trabajo, la informalidad, entre otras, que propician aspectos en común. Estas situaciones marcan estilos de liderazgo, establecimiento de políticas y estrategias de los directivos que en conjunto con los talentos y competencias de los integrantes de las organizaciones buscan alternativas para alcanzar la sostenibilidad empresarial.

En México, respecto a la responsabilidad social, hay entes reguladores, fundaciones y asociaciones que se dedican a desarrollarla en las empresas y a crear vínculos entre los trabajadores. En Colombia, las empresas se esfuerzan por brindar 
actividades de responsabilidad social a través de las personas; capacitaciones, actividades de bienestar. Está muy desarrollada la normativa en torno a la seguridad y la salud en el trabajo; se piensa en la protección del trabajador, en prevenir y mitigar los riesgos. Pero la responsabilidad social es más que cumplir con la norma, nace de un interés ético y genuino del querer proteger, brindar bienestar y cuidado a los colaboradores.

Gracias a las experiencias que brinda la Universidad de Ibagué, la docente Cecilia Eugenia Valdez Gutiérrez del Instituto Tecnológico de Monterrey pudo ser parte de la construcción del libro. Cecilia, además, ha participado en la Escuela Internacional de Verano. Es graduada en Ciencias de la Educación con especialización en Educación en Adultos. Tiene dos maestrías, una en Desarrollo Organizacional y otra en Educación para Adultos de la Universidad de Monterrey. Es doctora en Administración con especialidad en Recursos Humanos de Universidad de La Habana, Cuba. Ha dedicado sus esfuerzos en el desarrollo organizacional, recursos humanos, gestión humana y la responsabilidad social empresarial.

¿Qué aspectos hay que tener en cuenta para la construcción de prácticas con enfoque en responsabilidad social interna?

Con el libro descubrimos que hay diversidad de prácticas. No está escrito qué es y qué no es, hay una amplitud de actividades que se consideran parte de la responsabilidad social.

Figura 2. Retrato de Cecilia Eugenia Valdez Gutiérrez

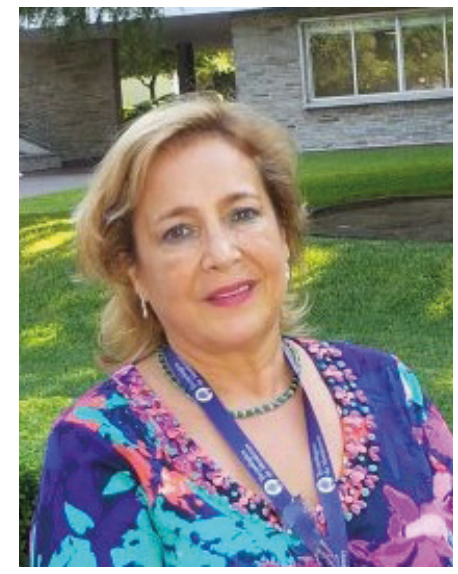

Fuente: Suministrada por la investigadora 
Sin embargo, no todos los programas gustan o motivan, porque las empresas tenemos diferentes generaciones con distintos intereses y valores. Así que, en primer lugar, atender a lo establecido en la ley. Los factores psicosociales son la primera guía en tomar decisiones. En segundo lugar, estudiar el tipo de población que existe en la organización y sus intereses. Hay que tener en cuenta los perfiles de los empleados, preguntarles sería lo ideal. La clave está en diseñar prácticas, pero teniendo en cuenta el contexto para que estas mismas sean exitosas.

\section{¿Cómo las organizaciones con una responsabilidad social fuerte pudieron enfrentar la pandemia?}

Las empresas nos tuvimos que vincular a la responsabilidad social interna de una manera muy rápida. Pero la cuestión está en que todos están exponiendo su vida, y el trabajador se tiene que sentir seguro. Se ha producido un movimiento a favor de la salud y seguridad del ser humano; empresa que no lo haga se queda sin gente. Esto ya no es negociable. La pandemia nos alineó, nos obligó a plantear más prácticas de responsabilidad social, puesto que, al no cumplir con la normatividad, se corre el riesgo de ser clausuradas. Esa va a ser la segunda parte del libro, vamos a ampliar en ¿qué prácticas durante la pandemia están implementando las empresas en términos de responsabilidad social? Ojalá podamos separar entre aquello que marca o no la ley, para ver la iniciativa de las organizaciones.

\section{¿Qué reflexiones trajo consigo la implementación del teletrabajo?}

Esto aceleró muchos cambios que venían. El teletrabajo era el cambio al que más le teníamos miedo en Latinoamérica, porque no confiábamos en el colaborador. Se tuvo que cambiar el sistema de administración, porque ¿cómo mido tu productividad?, ¿con clics, con cantidad de proyectos, con calidad? En algunos casos, este reto permitió que los equipos de trabajo se unieran aún más y ratificó identificar quiénes trabajan y quiénes no.

\section{Y respecto a la construcción del libro, ¿qué retos enfrentaron ustedes?}

La comparación fue el mayor reto, manejar las mismas variables comparativas y adquirir la misma información de empresas colombianas y mexicanas. Otro reto fue que no todas las empresas estuvieron abiertas a compartirnos la información, porque muchas veces, esas prácticas de responsabilidad interna son la fórmula secreta de las empresas para retener a su personal. 
Si bien es cierto que el área de gestión humana requiere de una alta inversión, la investigación resalta los beneficios y demuestra la importancia del establecimiento de planes y programas acordes con los intereses del personal. Lo anterior se presenta, puesto que, además de poner a prueba la creatividad e innovación de las organizaciones, el hecho de que un trabajador sienta que su empresa se preocupa por él es algo invaluable, y genera un mejor desempeño para la empresa con claridad.

\section{Referencias}

Vargas Montealegre, A. R., Valdez Gutiérrez,C.E., Díaz Valenzuela,S.,León Moreno, D.A., Rojas Herrera, D. A., \& Oviedo Bonilla, A. M. (2020). Prácticas sostenibles de Gestión Humana. Unénfasis desde la Responsabilidad Social Interna. Ibagué, Colombia: Ediciones Unibagué. https://doi.org/10.35707/9789587543407 